\title{
Scale separation between electron and ion thermal transport
}

\author{
T. Görler and F. Jenko \\ Max-Planck-Institut für Plasmaphysik, EURATOM Association, \\ Boltzmannstraße 2, D-85748 Garching, Germany
}

(Dated: 17 March 2008)

\begin{abstract}
Nonlinear gyrokinetic simulations of microturbulence simultaneously driven by electron temperature gradient modes, trapped electron modes, and ion temperature gradient modes are presented, covering both electron and ion spatio-temporal scales self-consistently. It is found that for realistic ion heat (and particle) flux levels and in the presence of unstable ETG modes, there tends to be a scale separation between electron and ion thermal transport. In contrast to the latter, the former may exhibit substantial or even dominant high-wavenumber contributions.
\end{abstract}

Usually, turbulent transport in magnetic fusion devices is thought to be carried mainly by long-wavelength modes with $k_{\perp} \rho_{s} \sim 0.2$. Here, $k_{\perp}$ is the perpendicular wavenumber and $\rho_{s}=c_{s} / \Omega_{i}$ where $c_{s}=\sqrt{T_{e 0} / m_{i}}$ is the ion sound speed and $\Omega_{i}$ the ion Larmor frequency. In this context, the main agents driving turbulent fluctuations in the core region are considered to be ion temperature gradient (ITG) modes and trapped electron modes (TEMs), while in the edge region, other microinstabilities like electron drift waves or resistive ballooning modes can also contribute [1]. However, there is strong experimental evidence that this standard paradigm cannot capture the behavior of heat transport in the electron channel in a number of important situations. These include, in particular, plasmas with internal or edge transport barriers. Here, the question arises which mechanism sets the residual anomalous electron thermal transport level inside a barrier, assuming that the turbulence at long wavelengths is suppressed by equilibrium $\mathbf{E} \times \mathbf{B}$ shear flows and that the ions are basically neoclassical [2-4]. Moreover, discharges with strong electron heating may also exhibit a decoupling of ion and electron heat transport which is best explained in terms of scale separation. E.g., in recent DIII-D experiments, it was found that by adding electron cyclotron resonance heating, the longwavelength dynamics (density fluctuation level) and the ion heat flux remain more or less unchanged while the short-wavelength dynamics at $k_{\perp} \rho_{s} \sim 4-10$ is strongly enhanced, accompanied by a substantial increase in the electron thermal diffusivity [5]. Thus, theory and simulation are confronted with assessing the role of the spectral region $k_{\perp} \rho_{s} \gg 0.2$, in particular for electron thermal transport.

Early gyrokinetic simulations of electron temperature gradient (ETG) turbulence - working mostly (although not exclusively) with the adiabatic ion approximation and neglecting magnetic trapping - indicated that despite their small spatio-temporal scales, ETG modes can induce electron thermal diffusivities which clearly exceed naive expectations, $\chi_{e} \gg \rho_{e}^{2} v_{t e} / L_{T_{e}}$, where $\rho_{e}$ is the electron thermal gyroradius, $v_{t e}$ is the electron thermal velocity, and $L_{T_{e}}$ is the electron temperature gradient scale length [6-8]. Thus, ETG turbulence has become a serious candidate for explaining experimental findings like the ones described above. In follow-up gyrokinetic work including magnetic trapping, this basic scenario was confirmed, but it also became clear that it is sometimes hard to find saturation in adiabatic ion models [9-12]. Moreover, one is even lead to question the validity of the resulting framework, since in the longwavelength limit, ETG modes often transition smoothly into TEMs which in turn extend down to the usual ion scales. However, these modes are not treated correctly in the adiabatic ion approximation - and ITG modes are excluded altogether. Consequently, what is really called for are nonlinear gyrokinetic simulations in which both electron and ion spatio-temporal scales are covered selfconsistently. Pioneering work along those lines was reported in Ref. [13], employing edge-like parameters and finding that ETG-induced electron thermal transport is capable of setting a base level in the H-mode pedestal region. Recently, there have been similar studies for core parameters, on the other hand, exhibiting only small relative high- $k$ contributions [14]. As we shall see below, the low- $k$ drive in these simulations has been unrealistically strong, however. In contrast to that, our results will show that for realistic ion heat (and particle) flux levels and in the presence of unstable ETG modes, there tends to be a scale separation between electron and ion thermal transport, i.e., the former may exhibit substantial or even dominant high- $k$ contributions.

The present work is based on massively parallel simulations with the gyrokinetic turbulence code GENE $[6,15]$. Although GENE is able to treat magnetic field fluctuations and collisions, these effects are neglected for simplicity here. Moreover, all simulations in this paper are performed in $\hat{s}-\alpha$ geometry with $\alpha=0$. Most physical parameters correspond to the so-called Cyclone base case (CBC) [16], i.e., we employ $q=1.4, \hat{s}=0.8, \epsilon=r / R=$ $0.18, n_{i} / n_{e}=1$, and $T_{i} / T_{e}=1$. The density and temperature gradients deviate from the $\mathrm{CBC}$, however, and will be stated on a case-by-case basis. The perpendicular box size is chosen to be $\left(L_{x}, L_{y}\right)=\left(64 \rho_{s}, 64 \rho_{s}\right)$, and we use $768 \times 384 \times 16$ grid points in the radial, binormal, and parallel direction, respectively, as well as $32 \times 8$ grid points in $\left(v_{\|}, \mu\right)$ space. Two particle species (ions and 


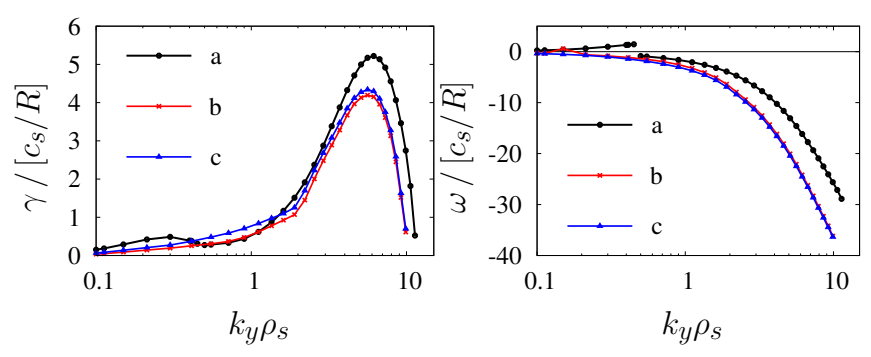

FIG. 1: Linear growth rates (left) and real frequencies (right) vs. binormal wavenumber using (a) $R / L_{T_{i}}=R / L_{T_{e}}=6.9$, $R / L_{n}=2.2$, (b) $R / L_{T_{i}}=5.5, R / L_{T_{e}}=6.9, R / L_{n}=0$, and (c) $R / L_{T_{i}}=0, R / L_{T_{e}}=6.9, R / L_{n}=0$.
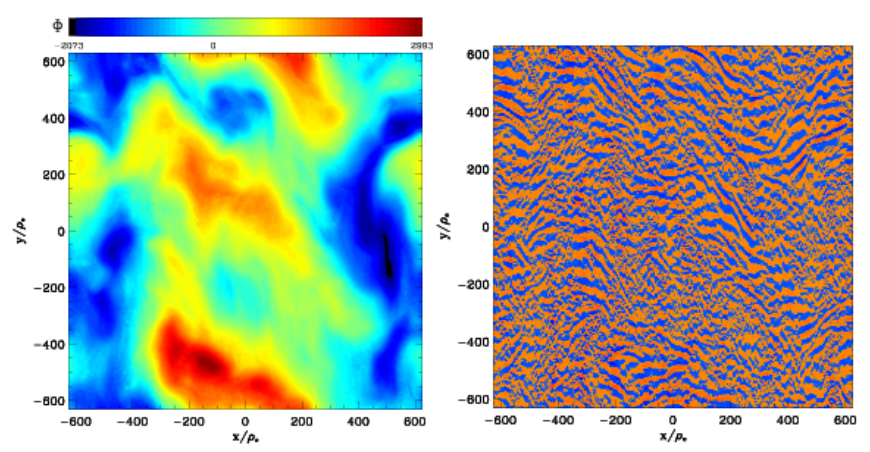

FIG. 2: Snapshot of the electrostatic potential for $R / L_{T_{i}}=$ $R / L_{T_{e}}=6.9$ and $R / L_{n}=2.2$, displaying a dominance of large-scale, isotropic ITG vortices, and the same data with all $k_{y} \rho_{s}<2$ modes filtered out, exhibiting the existence of smallscale ETG streamers which are subject to vortex stretching.

electrons) are kept, both of them fully gyrokinetic. Since the computational effort roughly scales like $\left(m_{i} / m_{e}\right)^{3 / 2}$ (due to scale separation in $x, y$, and $t$ ), we will work with a reduced mass ratio of $m_{i} / m_{e}=400$; still, each simulation requires of the order of 100,000 CPUh.

In a first run, we choose $R / L_{T_{i}}=R / L_{T_{e}}=6.9$ and $R / L_{n}=2.2$, in line with the nominal $\mathrm{CBC}$ values. The resulting $k_{y}$ spectra of the linear growth rates and real frequencies are shown in Fig. 1 as the curves with the label (a). Up to a binormal wavenumber of $k_{y} \rho_{s} \sim 0.4$, ITG modes (identified via the sign of the real frequency) dominate, whereas at smaller scales, TEMs have the largest growth rate, smoothly transitioning into ETG modes at $k_{y} \rho_{s} \sim 1$.

Corresponding nonlinear simulation results are shown in Figs. 2 and 3. While a first glimpse at a snapshot of the electrostatic potential on the low-field side shown in Fig. 2 suggests that the large-scale ITG dynamics dominates, a filtered image reveals that short-wavelength structures co-exist with the large-scale vortices. In order to assess the contribution of these strongly distorted ETG streamers to the overall (electron) heat transport, time-averaged spectra of the ion and electron thermal diffusivities are presented [in units of $\chi_{\mathrm{gB}}=c_{s} \rho_{s}^{2} / R$ ] in Fig. 3, using a somewhat unconventional but helpful way
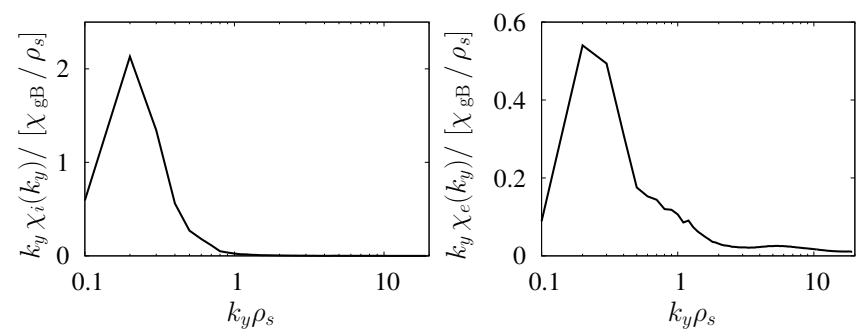

FIG. 3: Time-averaged ion (left) and electron (right) thermal heat diffusivities multiplied by binormal wavenumber vs wavenumber for $R / L_{T_{i}}=R / L_{T_{e}}=6.92, R / L_{n}=2.2$.

to display the data. While the usual log-log plots can be misleading since the area underneath a curve has no direct physical meaning, plotting $k_{y} \chi\left(k_{y}\right)$ vs. $k_{y}$ on a log-lin scale circumvents this problem. In contrast to pure ITG or TEM simulations (with ETG modes linearly stable), where both thermal diffusivity spectra tend to peak at $k_{y} \rho_{s} \sim 0.2$ and fall off quickly with $k_{y}$, a relatively small but finite fraction of $10.5 \%$ of the total $\chi_{e}\left[\sim 8.1 \chi_{\mathrm{gB}}\right]$ originates at $k_{y} \rho_{s}>1$. This is in good agreement with simulation results presented in [12] where a high- $k$ contribution of $13.8 \%$ has been found using the same physical parameters.

In order to correctly interprete these findings, it is necessary to compare the above quantities with the experimental ones, using the plasma parameters underlying the $\mathrm{CBC}$ values employed here. This way, one finds that the ion thermal diffusivity obtained from the simulation, $\chi_{i}^{\text {sim }} \sim 23.7 \chi_{\mathrm{gB}}$, exceeds the experimentally determined value of $\chi_{i}^{\exp } \sim 0.36 \chi_{\mathrm{gB}}$ [16] by almost two orders of magnitude. The most likely reason for this dramatic difference is that the normalized ion temperature gradient $R / L_{T_{i}}$ - on which ITG turbulence depends very strongly but whose extraction from experimental temperature profile data is usually difficult - has been chosen somewhat too large.

This motivates us to perform a second simulation with the same parameters as before except for $R / L_{n}=0$ and $R / L_{T_{i}}=5.5$, which is still about $10 \%$ above the linear ITG threshold of $R / L_{T_{i}}^{\text {crit }} \approx 5$.0. Using these new parameters, one obtains the linear growth rates and real frequencies shown in Fig. 1 as the curves with the label (b). Now, a combined trapped electron and ETG mode dominates over (almost) the entire $k_{y}$ range, but an ITG mode co-exists in the low- $k_{y}$ region (up to $k_{y} \rho_{s} \sim 0.5$ ), exhibiting a growth rate which is roughly comparable to that of the TEM.

Nonlinearly, one finds that for $k_{y} \rho_{s} \lesssim 1$, the turbulence displays a clear ITG-type character, whereas for larger wavenumbers, it is ETG-like. This statement is based, among other things, on comparisons of nonlinear frequencies and cross phases (see Fig. 4b) with the respective linear quantities. Furthermore, snapshots of the 

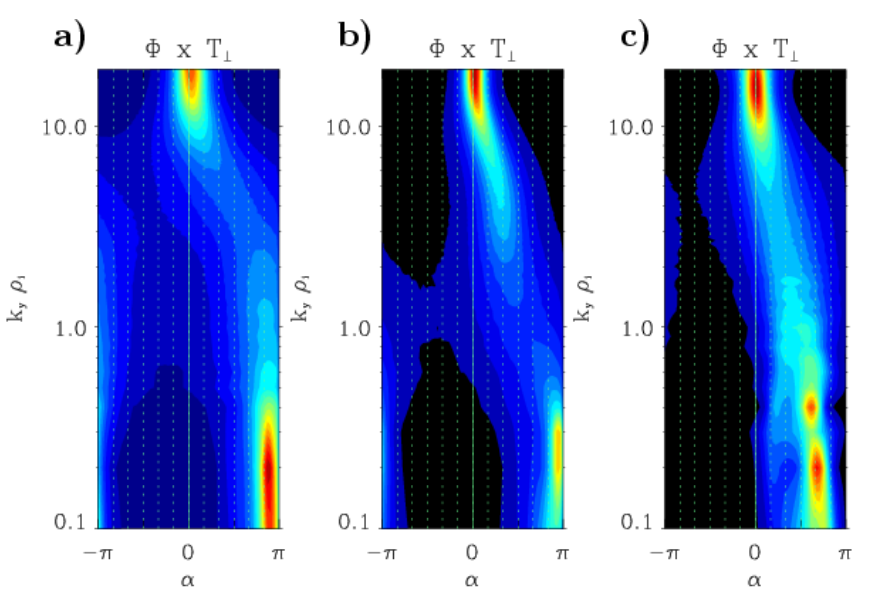

FIG. 4: Cross phases between electrostatic potential and perpendicular electron temperature against phase angle $\alpha$ and binormal wavenumber $k_{y}$ for (a) $R / L_{T_{i}}=R / L_{T_{e}}=6.9$, $R / L_{n}=2.2$, (b) $R / L_{T_{i}}=5.5, R / L_{T_{e}}=6.9, R / L_{n}=0$, and (c) $R / L_{T_{i}}=0, R / L_{T_{e}}=6.9, R / L_{n}=0$.

electrostatic potential look quite similar to those shown in Fig. 2. However, thermal diffusivities and the corresponding $k_{y}$ spectra change dramatically. Most importantly, the ion thermal diffusivity drops by almost one order of magnitude, reaching a value of $\chi_{i} \sim 2.9 \chi_{\mathrm{gB}}$, while the corresponding $k_{y}$ spectrum remains qualitatively unchanged, except for a slight shift of the transport peak to higher wavenumbers. In addition, the total electron thermal diffusivity is decreased by a similar factor, reaching $\chi_{e} \sim 0.8 \chi_{\mathrm{gB}}$. This change is mainly due to the large ITG-dominated scales (which exhibit a significant $\chi_{e}$ component) and less pronounced at high $k_{y}$. In the latter regime, the cross phase between $\phi$ and $T_{e, \perp}$ (these quantities are considered since it turns out that most of the electron thermal transport is carried by the term $\left.\left\langle T_{\perp} v_{E x}\right\rangle\right)$ reaches values close to $\pi / 2$ for $2 \lesssim k_{y} \rho_{s} \lesssim 5$ (cp. Fig. 4a and b), thus more or less compensating the amplitude reduction. Consequently, the high- $k$ fraction increases substantially as can be observed in Fig. 5. Now, more than $50 \%$ of the electron thermal transport originates from wavenumbers with $k_{y} \rho_{s}>0.5$, which is completely different from pure ITG or TEM turbulence simulations (with ETG modes being linearly stable) where typically less than $10 \%$ is carried by modes in this range. Therefore, the present case - which might be interpreted as a plasma with strong electron heating - is subject to a scale separation between the ion and electron heat fluxes. While the ion channel only displays contributions from $k_{y} \rho_{s}<1$, the electron channel may exhibit substantial high- $k$ contributions driven by ETG modes.

Since the simulation results obviously depend very strongly on $R / L_{T_{i}}$, we also perform a simulation in which just TEMs and ETG modes are driving the turbulence. This may happen, e.g., in plasmas with dominant electron heating, relatively high beta values, substantial equi-
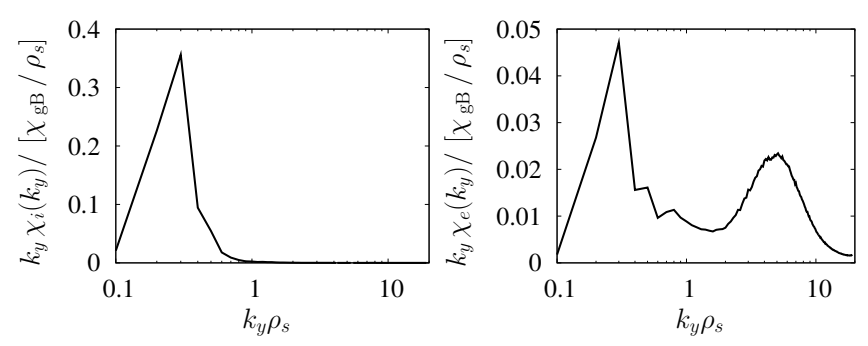

FIG. 5: $k_{y}$ spectra of the time-averaged ion (left) and electron (right) thermal fluxes for $R / L_{n}=0, R / L_{T_{i}}=5.5$, and $R / L_{T_{e}}=6.9$. For these parameters, a scale separation between both channels is observed.
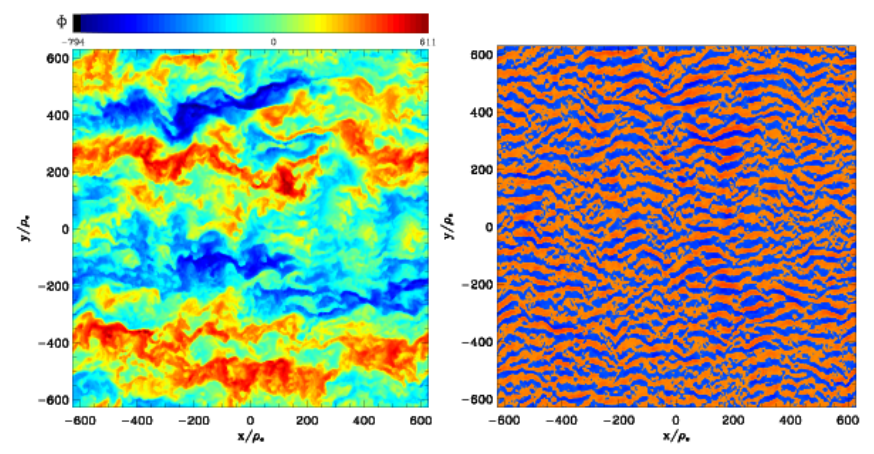

FIG. 6: Potential contour at low-field side for $R / L_{T_{i}}=0.0$ and $R / L_{T_{e}}=6.9$ and the same contour neglecting all modes $k_{y} \rho_{s}<2$.

librium $\mathbf{E} \times \mathbf{B}$ shear, or internal transport barriers. The profile gradients for this simulation are chosen to be $R / L_{T_{e}}=6.9$ and $R / L_{T_{i}}=R / L_{n}=0.0$. In this context, it might be useful to note that the choice of $R / L_{T_{i}}$ should not matter too much as long as ITG modes are (linearly and also nonlinearly) clearly subdominant, and that $R / L_{n}$ should be chosen small enough, such that the TEM is $\nabla T_{e}$-driven, not $\nabla n$-driven (in the latter case, one would obtain large outward particle fluxes which are usually hard to reconcile with experimental conditions).

The respective linear properties are presented in Fig. 1 as the curves with the label (c). Whereas in the high- $k$ part of the spectra, there is no qualitative change, the $k_{y} \rho_{s}<1$ range is now dominated by TEMs, of course. Both modes transition into each other smoothly, i.e., they belong to the same root and there is no jump in the real frequency. The nonlinear simulation reveals a completely different behavior compared to that with low- $k$ ITG turbulence. Instead of isotropic vortices, the contour plots of the electrostatic potential exhibit radially elongated structures with a multitude of different length scales, as can be seen in Fig. 6. This streamer-like behavior is in line with previous (pure) ETG and TEM simulations $[6,15]$. A comparison of the filtered images in Figs. 2 and 6 suggests that the medium- $k$ and high- $k$ fluctuations are less affected by the large-scale fluctuations than in the ITG case. This statement is supported by the 
good agreement of the electron thermal diffusivities in simulations restricted to $k_{\perp} \rho_{s}>1$ [8] and in the current multi-scale simulation, evaluated at $k_{y} \rho_{s}>1$. The corresponding $k_{y}$ spectrum is shown in Fig. 7. In contrast
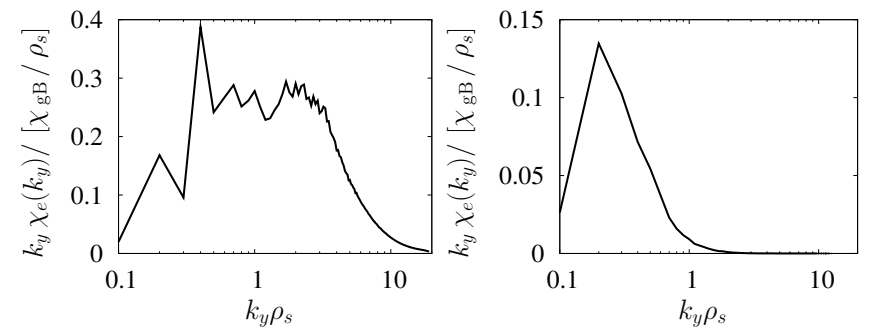

FIG. 7: $k_{y}$ spectra of the electron thermal diffusivity for TEM-ETG multiscale turbulence simulation (left) and a pure TEM turbulence simulation where ETG modes are linearly stable (right).

to pure TEM or ETG turbulence simulations, where one finds that the transport spectra are usually localized in fairly narrow regions of $k_{y}$ space (see, e.g., Fig. 7 and Ref. [8]), here, a wide range of modes contributes significantly to the overall thermal diffusivity of $\chi \sim 8.82 \chi_{\mathrm{gB}}$. About $30 \%$ of the transport is driven in the "classical" TEM range, $k_{y} \rho_{s} \lesssim 0.5$. The remaining high- $k$ contribution can be divided into a TEM-ETG turbulence region up to $k_{y} \rho_{s}<2$ (at this point, the trapped electron bounce frequency matches approximately the mode frequency) and an ETG region at $k_{y} \rho_{s}>2$. The thermal transport fraction produced in the latter region is almost equal to that of the low- $k$ region, namely about $30 \%$. However, the electron thermal diffusivity of the present TEM-ETG turbulence simulation is higher compared to the heat diffusivity gained with $R / L_{T_{i}}=5.5$, thus diverging from experimental values [10] again. One reason for this behavior is that the cross phases between $\phi$ and $T_{e, \perp}$ get close to $\pi / 2$ over a wide region in $k_{y}$ space as can be seen in Fig. 4c, leading to an increased transport level at intermediate and high wavenumbers. Obviously, this scenario deviates fundamentally from the ITG-TEMETG case where nonlinearly, ITG features dominate up to $k_{y} \rho_{s} \sim 1$, thus wiping out the intermediate-scale TEM dynamics and also reducing the ETG fluctuations. This can be viewed as a violation of the superposition principle in plasma microturbulence. Different types of modes/turbulence, in general, should not be seen as additive, but rather as competitive, as suggested, e.g., in the theoretical work by Itoh and co-workers [17-19].

In conclusion, we have shown in this paper by means of nonlinear gyrokinetic simulations covering both electron and ion spatio-temporal scales self-consistently that for realistic ion heat (and particle) flux levels and in the presence of unstable ETG modes, there tends to be a scale separation between electron and ion thermal transport. In contrast to the latter, the former may exhibit substantial or even dominant high-wavenumber contributions carried by ETG modes and short-wavelength TEMs which are only relevant nonlinearly if ETG modes are unstable. Thus, ETG modes can influence the transport spectrum over a surprisingly wide range, down to $k_{y} \rho_{s} \sim 0.5$ in the cases studied here. This effect can be further enhanced in situations where ITG modes are subdominant or (nonlinearly) stable like, e.g., in plasmas with dominant electron heating, relatively high beta values, substantial equilibrium $\mathbf{E} \times \mathbf{B}$ shear, or internal transport barriers. Here, the long-wavelength ITG contributions to the total heat flux can be small or even negligible - as has been shown in many previous studies and the high-wavenumber TEM/ETG contributions will be even more pronounced. Simulations for specific fusion devices with more complete physics are left for future work.

We would like to thank F. Merz for valuable discussions. The computations were performed at LeibnizRechenzentrum, Garching.

[1] P. C. Liewer, Nucl. Fusion 25, 543 (1985).

[2] J. W. Connor and H. R. Wilson, Plasma Phys. Controlled Fusion 42, R1 (2000).

[3] R. C. Wolf, Plasma Phys. Control. Fusion 45, R1 (2003).

[4] T. S. Hahm et al., Phys. Plasmas 12, 090903 (2005).

[5] T. L. Rhodes et al., Phys. Plasmas 14, 056117 (2007).

[6] F. Jenko et al., Phys. Plasmas 7, 1904 (2000).

[7] Dorland et al., Phys. Rev. Lett. 85, 5579 (2000).

[8] F. Jenko and W. Dorland, Phys. Rev. Lett. 89, 225001 (2002).

[9] Y. Idomura, Phys. Plasmas 13, 080701 (2006).

[10] W. M. Nevins et al., Phys. Plasmas 13, 122306 (2006).

[11] A. Bottino et al., Phys. Plasmas 14, 010701 (2007).

[12] J. Candy et al., Plasma Phys. Control. Fusion 49, 1209 (2007).

[13] F. Jenko, J. Plasma Fusion Res. SERIES 6, 11 (2004).

[14] R. E. Waltz, J. Candy, and M. Fahey, Phys. Plasmas 14, 056116 (2007)

[15] T. Dannert and F. Jenko, Phys. Plasmas 12, 072309 (2005).

[16] A. M. Dimits et al., Phys. Plasmas 7969 (2000).

[17] S.-I. Itoh and K. Itoh, Plasma Phys. Control. Fusion 43 1055 (2001).

[18] S.-I. Itoh et al., Plasma Phys. Control. Fusion 441311 (2002).

[19] K. Itoh et al., Plasma Phys. Control. Fusion 45911 (2003). 